Claremont Colleges

Scholarship@ Claremont

Pomona Faculty Publications and Research

Pomona Faculty Scholarship

$1-1-2012$

\title{
Medicine, Statistics, and Education: The Inextricable Link
}

Katharine K. Brieger'11

Pomona College

Johanna S. Hardin

Pomona College

\section{Recommended Citation}

Brieger, K.†, and Hardin, J. Medicine and Statistics: the inextricable link, Chance, 25: 31-34; 2012.

This Article is brought to you for free and open access by the Pomona Faculty Scholarship at Scholarship @ Claremont. It has been accepted for inclusion in Pomona Faculty Publications and Research by an authorized administrator of Scholarship @ Claremont. For more information, please contact scholarship@cuc.claremont.edu. 


\section{Medicine, Statistics, and Education: The Inextricable Link}

Katharine Brieger and Johanna Hardin

tatistics is an integral part of any clinical trial, and it is widely recognized that the training of doctors should aim to improve statistical literacy. However, the call for statistical literacy has focused on introductory material, and we argue that there is a need for advanced statistical literacy in the health sciences (Table 1).

In their 2005 article, "Statistical Methods in the Journal," Nicholas Horton and Suzanne Switzer reviewed all New England Journal of Medicine articles from January 2004 to June 2005; they found that more than half of the articles used survival analysis, multiple regression, or another advanced technique. A 2007 study appearing in the Joumal of the American Medical Association looked at residents' understanding of statistics; authors Donna Windish, Stephen Huot, and Michael Green showed that only 10.5\% knew how to interpret the results of a Kaplan-Meier curve and only $37.4 \%$ could interpret an adjusted odds ratio from a multiple regression.

The need for extended statistical education for our health scientists is greater than previously imagined.

Recently, the 'dangers' of the vaccine for measles, mumps, and rubella (MMR) made headlines in the United Kingdom, warning parents about the vaccination's associated risk for autism. The alarm was based on an article appearing in The Lancet, Andrew Wakefield's 1998 study on 12 children who had developmental regression. Given high rates of both MMR vaccination and autism, finding 12 children who had received the MMR vaccine and also had autism was unremarkable. Additionally, the symptoms of autism are often first noticed at the same age as vaccination, so the relationship may have been coincidental, not causal.

In 2004, The Lancet retracted Wakefield's article, but its effect has been particularly damaging to communities in which measles, mumps, and rubella were previously all but eradicated. In the United Kingdom in 2003-2004, the MMR vaccination rate reached an all-time low of $80 \%$, much below the herd immunity rate of $95 \%$. In 2008, measles was declared endemic to England and Wales for the first time since 1984.

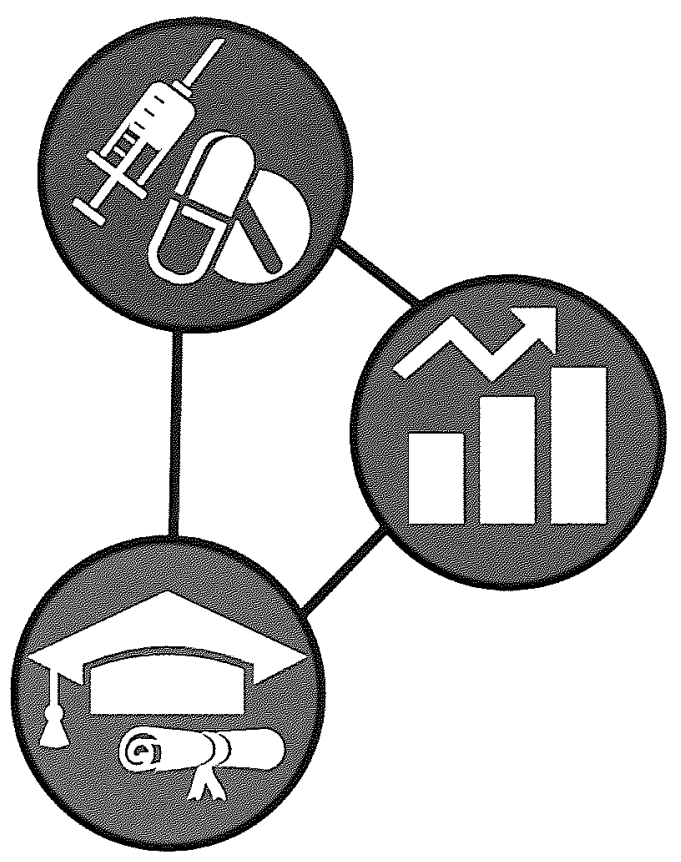

This example illustrates how misinterpretation of statistical results can have serious public health consequences. Basic knowledge of observational study limitations, variability, and small sample sizes should have prevented the unwarranted conclusion that the MMR vaccine causes autism. However, we would take this example a step further and claim that additional statistical knowledge (e.g., on multiplicities) could have further reduced the chances of the misinterpretation of the Wakefield paper.

\section{Table 1-Top Five Most Commonly Used Statistical Methods in New England Journal of Medicine Articles Across Time}

\begin{tabular}{|c|c|c|}
\hline $\begin{array}{c}\text { Then } \\
(1978-1979)\end{array}$ & Now (2005) & Soon to Come \\
\hline $\begin{array}{ll}\text { - } & \text { tests }(44 \%) \\
\text { - } & \text { contingency } \\
\text { tables }(27 \%) \\
\text { - } & \text { Pearson correla- } \\
\text { tion }(12 \%) \\
\text { - nonparametric } \\
\text { tests }(11 \%) \\
\text { survival meth- } \\
\text { ods }(11 \%)\end{array}$ & $\begin{array}{ll}\text { - } & \text { survival meth- } \\
\text { ods }(61 \%) \\
\text { - } & \text { contingency } \\
\text { tables }(53 \%) \\
\text { - } & \text { multiple regres- } \\
& \text { sion }(51 \%) \\
\text { - } & \text { power analysis } \\
& (39 \%) \\
\text { - } & \text { epidemiologic } \\
& \text { statistics }(35 \%)\end{array}$ & $\begin{array}{l}\text { multiple com- } \\
\text { parisons } \\
\text { analysis of high- } \\
\text { throughput data } \\
\text { - advanced } \\
\text { experimental } \\
\text { design } \\
\text { - Bayesian } \\
\text { analysis } \\
\text { permutation/ } \\
\text { bootstrap } \\
\text { methods }\end{array}$ \\
\hline
\end{tabular}

First two columns from Switzer and Horton's "What Your Doctor Should Know About Statistics (but Perhaps Doesn't), "CHANCE, 2007. Percent of papers using the methodology is given in parenthesis. Last column is a prediction of methods that are quickly growing in popularity. 
In a provocative 2005 article (with a provocative title, "Why Most Published Research Findings Are False"), John loannidis demonstrates that under certain typical conditions, most published research findings are false. To understand the findings, we first consider the sel-up of a typical experiment in a particular field of science. Of course, there is not any static field of science, but we imagine a given set of all hypothesis tests done with that field.

Using the notation from loannidis, let $c$ be the total number of tests in the field, and let $R$ represent the ratio of the number of "true relationships" to "null relationships" among those tested in the field. Note that $R$ is typically unknowable, and we differentiate between "true" relationships and "significant" relationships; $R$ considers the former. Additionally, assume the standard notation for the probability of making a type I error (rejecting a null relationship, $\alpha$ ) and the probability of making a type $\mathrm{II}$ error (not rejecting a true relationship, $\beta$ ).

Using the identity: \# true relationships $=R^{*}$ (\# null relationships), we can calculate the proportion of true relationships.

proportion of all tests that are true relationships $=R /(R+1)$

The number of true and null relationships is simply the appropriate proportions of the total, $c$, number of tests.

$$
\begin{aligned}
& \# \text { of true relationships }=c \frac{R}{R+1} \\
& \# \text { of null relationships }=c \frac{1}{R+1}
\end{aligned}
$$

Using type I and type II error rates, we can find the number of significant relationships out of those that are true and the number of significant relationships out of those that are null.

$$
\begin{gathered}
\text { \# of relationships that are significant and true }=c \frac{R}{R+1}(1-\beta) \\
\text { \# of relationships that are significant and null }=c \frac{1}{R+1} \alpha
\end{gathered}
$$

All significant relationships are given by the sum of those from the true and null groups.

$$
\# \text { of significant relationships }=\frac{c}{R+1}(R(1-\beta)+\alpha)
$$

Finally, the positive predicted value (PPV) is calculated as the proportion of true studies out of those that are reported to be significant.

$$
P P V=\# \text { of true studies } \mid \text { significance }=\frac{R(1-\beta)}{R(1-\beta)+\alpha}
$$

The PPV gives a measure of what is desired in science: the probability of a true relationship given a significant result. Note that PPV $>0.5$ (a significant result is more likely true than false) only if $(1-\beta) R>\alpha$.

\section{Modern Statistical Challenges}

\section{Multiplicities}

We define multiplicities to mean multiple tests of hypotheses on the same data-many variables, multiple endpoints to a study, multiple time points, comparing several treatments, interim analyses, considering an endpoint as continuous or categorical. The prevalence and impact of multiplicities is often underestimated, and there is evidence for a large proportion of false findings in the current literature.

In a controversial 2011 study, Daryl Bem tested college students for precognition and premonition abilities, types of extrasensory perception (ESP). Although he obtained highly significant results, some point to Bem's research as an example of the problems with multiple comparisons. Although the majority of Bem's ESP experiments were significant, they represent a small fraction of all ESP experiments that have been done, most of which are not published.

Interestingly, the degree of association in the ESP research is much stronger than that in the MMR case; however, the acceptance of the research, itself, is reversed. The contrast in the reactions to the significance of these studies indicates a need for understanding the process used to arrive at the results. The experimental design, statistical analysis, and prior assumptions related to having a true relationship are all components of how likely a research statement is to be true.

Using the results from John Ioannidis in the sidebar, we can assess the MMR and ESP examples. Keep in mind that, ideally, we would be able to evaluate our work using the probability of a true relationship given a significant result, as opposed to the standard hypothesis testing structure in which we constrain the probability of a significant result given a null relationship. The positive predictive value (PPV) measures the proportion of true relationships out of those found significant; we should hope for a large PPV for all of our work.

It is worth considering the values of $R$ (the ratio of true relationships to null relationships of all those tested in the field) and $\beta$ (type II error rate) in each of the studies. One might have a prior belief that $\mathrm{R}$ is particularly low with ESP. Additionally, with the small sample size in the MMR study, $\beta$ would be particularly high. Low $\mathrm{R}$ and high $\beta$ give rise to a small PPV, and thus the results of such studies should be considered carefully. Ioannidis extends his analysis to include situations with bias and multiple independent research teams working on the same problem. A standard type of bias is to use some form of multiplicity - variable selection, change in endpoint, etc. Each statistical analysis pushed toward significance via some form of multiplicities induces a lower PPV.

The issues of multiplicities can be ameliorated with validation studies and a more thorough understanding of variability across the system at hand. Purely chance findings are often published and mistakenly 
considered important simply because positive results are more likely to be published. We need to use our statistical knowledge not only to assess the data, but also to evaluate the merit of published results. Medical education would greatly benefit from a second course in statistics that better prepared doctors to interpret results for themselves.

\section{Large Data Sets}

High-throughput data-as from microarray, proteomic, and next generation sequencing-have become ubiquitous and invaluable in medical research. However, unlike many statistical techniques applied in the medical literature, methods used to analyze high-throughput data are sophisticated and not taught in standard statistical curricula. Additionally, high-throughput data are prone to measurement and preprocessing errors.

We present the 2009 work of Keith Baggerly and Kevin Coombes, published in Annals of Applied Statistics, in what they term "forensic bioinformatics." In 2007, Duke University initiated a series of clinical trials based on analyses from microarray studies that modeled genetic characteristics predictive of sensitivity to certain drugs. The initial work by Anil Potti and colleagues, published in Nature Medicine in 2006, was retracted after patients were enrolled in three different clinical trials that were subsequently suspended in June of 2010.

The underlying problem with high-throughput data is lack of intuition about how certain markers or tests should behave, particularly diagnostic procedures based on tens or hundreds of genetic markers. It is difficult to make relevant predictions based on data that are so large they cannot be conceptualized. Because analysis of high-throughput data is typically exploratory, simple mistakes are easy to make, but difficult to discover.

In the Duke research, simple mistakes led to the complete invalidity of their results. The experimental conditions were confounded with the date of the experiments, making any differences in conditions unknowable. A gene label mix-up created a model that included genes that had not been measured to predict sensitivity to chemotherapy agents. The outcome labels were switched on the microarrays for a group of patients, thus creating a model predicting exactly the opposite treatment than that which should have been applied.

The difficulties with large and high-throughput data sets are not all simple and technical. To quantify genetic activity from microarray data, image-processing techniques are applied to a scan of a biological sample labeled with florescent dye and hybridized to a chip. Additional algorithms adjust the numerical values to account for systematic changes (e.g., dye biases, edge effects, heteroskedasticity). The choice of image processing or preprocessing algorithms and parameters necessarily affect the numerical value outcomes that represent genetic activity. The statistical training required to understand microarray analyses is much more thorough than a conventional introductory curriculum.
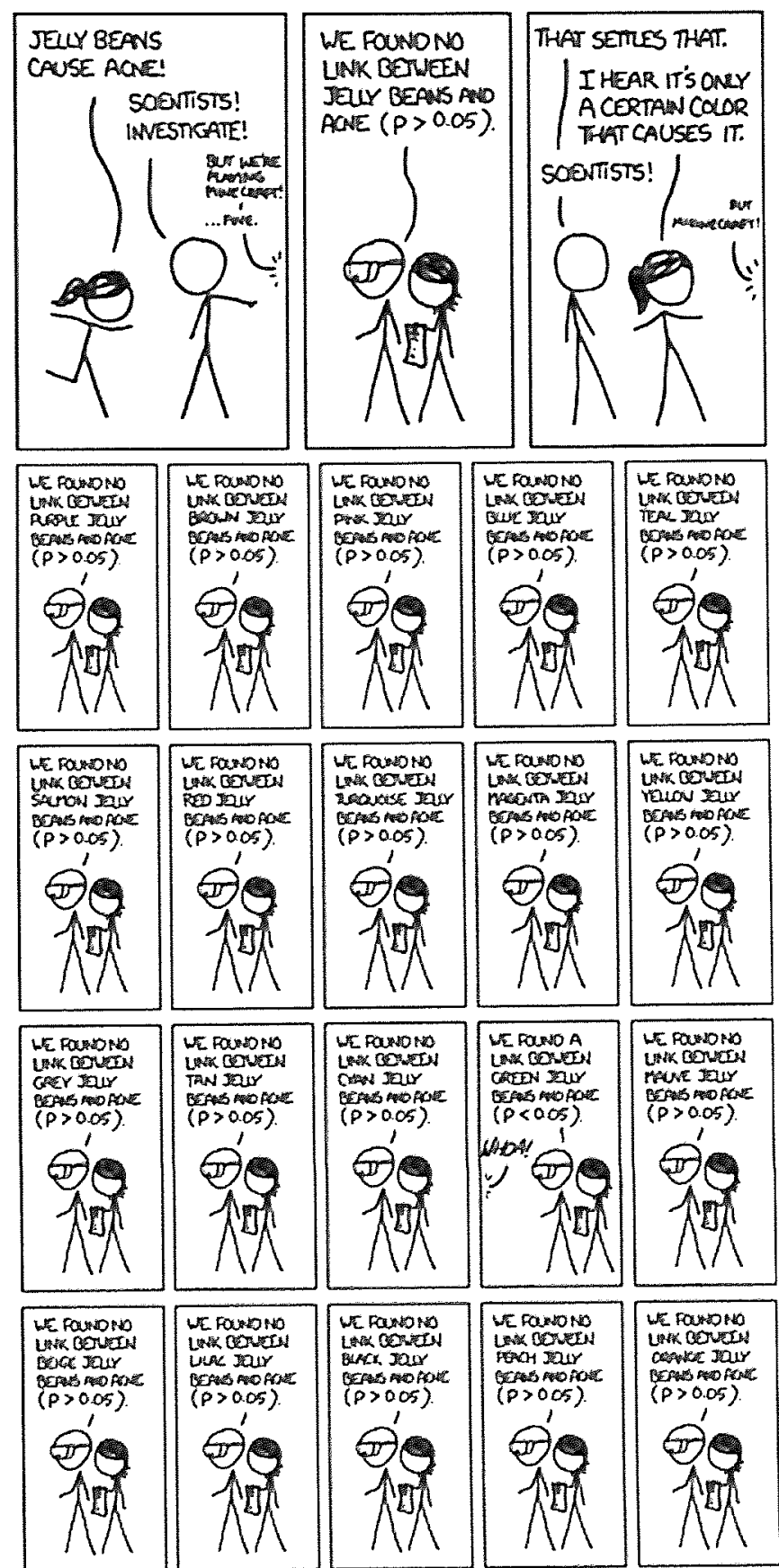

WC Paw to Letrover con $(\rho>0,0)$.
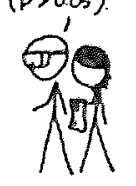

$\checkmark \cos 0 \mathrm{~A}$ Lotos cover cenos notar $(p<005)$. (c)

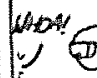
Wo

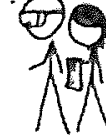

Lepowion recues sur

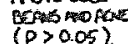

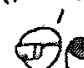

W Kanots Laverou gencr. Jan $(\rho>0,5)$ $(P>005)$
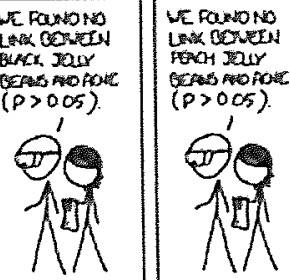
LAs nover Ders jear $(p>0,1)$.

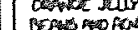
$(p>0.05)$

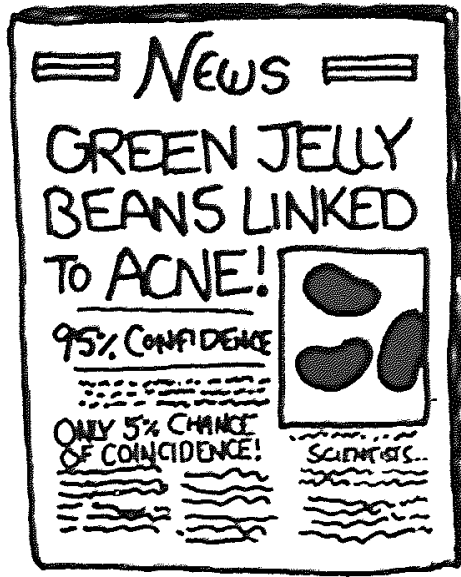

COMIC COURIESY OF HITP://XKCD.COM/882 


\section{Experimental Design}

Not every clinical trial is straightforward with 1:1 randomization between two treatments; researchers use historical controls, inverse sampling, and adaptive randomization. Though there are benefits to each design, trials using advanced experimental designs are difficult to analyze. From the outset, it is imperative to state the explicit design structure to control the type I error rate accurately. Additionally, later statistical analyses must take into account any unusual data structures.

One alternative to $1: 1$ randomization is adaptive randomization, randomizing more patients to the treatment arm that shows the best prognosis. In the 1980s, a series of trials was conducted to ascertain whether extracorporeal membrane oxygenation (ECMO) was effective for treatment of persistent pulmonary hypertension of the newborn. At the time, there was mounting evidence that ECMO was superior to conventional therapy, and there were ethical concerns over implementing a standard randomized design to demonstrate ECMO's efficacy.

Robert Bartlett and colleagues conducted an initial adaptive design experiment in 1985; only one patient was given conventional treatment, and that patient died. Though statistically significant, the small sample size was unconvincing to the medical community, warranting further study.

In 1989, James Ware designed a two-stage clinical trial in which treatments were selected using permutedblock randomization. The study eventually enrolled 30 patients with the ECMO treatment, 25 of whom survived. All nine patients enrolled in the conventional treatment died. According to Ware's Statistical Science piece, "Investigating Therapies of Potentially Great Benefit: ECMO," as compared to standard 1:1 randomization, the creative study design subjected fewer patients to the less efficacious conventional treatment.

The decision to implement an adaptive design is not always straightforward. Often, the motivation for an adaptive design is to create a setting in which fewer patients are subjected to the treatment arm that is substantially worse. In 2011, though, Edward Korn and Boris Freidlin demonstrate through simulations

\section{About the Authors}

Kotharine Brieger graduated from Pomona College with a BA in environmental analysis and a particular interest in environmental determinants of health. She is currently combining her passion for statistics and medicine through her sludies in the MD-PhD program at the University of Michigan. She is pursuing the $\mathrm{PhD}$ in biostatistics.

Jo Hardin is an associate professor at Pomona College. Her research interests include outlier detection, correlation metrics, clustering, and robust methods, particularly applied to high-throughput data sets. that the sample sizes needed to power an adaptive study lead to more patients on the "worse" arm of treatment than standard randomization would, although they are a smaller proportion of total subjects. However, in their article, "Outcome-Adaptive Randomization: Is It Useful?" Korn and Freidlin neglect to mention the patients who are not enrolled in the study, but do have the disease. Those patients also must be treated, so the effective number of patients given the worse treatment is likely still less in the adaptive setting.

Though adaptive designs may be well suited for situations with large differences (as with ECMO) or multi-arm trials, they also bring with them a host of logistical complications that can undermine the actual adaptation. Collecting data in a timely manner from multiple sites is not trivial, and we should be wary to think that the theoretical justification trumps other concerns in designing studies that will communicate maximal information to the medical community.

\section{Conclusion}

In 1937, an article in The Lancet criticized physicians' "blind spot" in laboratory and clinical medicine to be simple statistical methods. In 1948, the British Medical Association recommended that statistics be included in medical education. Yet, for example, it was not until 1975 that statistics became mandatory at the University of London School of Medicine. In 2009, the Association of American Medical Colleges listed among its recommended competencies for medical school graduates the ability to "apply quantitative knowledge and reasoning-including integration of data, modeling, computation, and analysis-and informatics tools to diagnostic and therapeutic clinical decisionmaking."

Increasingly, many of the important statistical considerations in medical studies are far beyond the knowledge gained in introductory statistics; we must remember that just as medicine is a dynamic and fastmoving field, so is statistics. As we keep abreast in both fields simultaneously, we enhance our ability to expand upon knowledge of human health. $\mathbf{E}$

\section{Further Reading}

Altman, D.G., and J.M. Bland. 1991. Improving doctors' understanding of statistics. J $R$ Statist Soc $A$ 154: 223-267.

Berry, Donald A. 2011. Adaptive clinical trials: the promise and the caution. Journal of Clinical Oncology 29(6):606-609.

Mathematics and Medicine. 1937. Editorial, The Lancet 31(1). 